\title{
Visual Prognosis and Surgery in Tuberculum Sellaemeningiomas
}

\author{
k.BOUAITA,L.ATROUNE SHABAY.Z, N.IOUALALEN \\ Department of neurosurgery, hospital ali ait idir, university of algeries, algeria
}

\begin{abstract}
They are benign tumors of which surgical excision should be total so as to avoid recurrences. The operative difficulties rests in the lose relationship between the tumors and major arteries of the anterior part of the circle of Willis in one part and with the optic pathways and the pituitary stalk in the other part. Clinical symptoms are dominated by the optochiasmatic syndrome with visual disturbances in $95 \%$ of patients.

We report a series of 64 cases, who have been operated on at Ali Ait Idir Hospital in Algiers, during a period of 10 years.

$=62$ patients have been operated on by transcranial approach:

Unilateral Subfrontal approach in 15 cases

Fronto temporal approach in 35 cases ;

Subfrontal with supra orbital incision in 12 cases

$=$ One patient has been operated on by endoscopic transsphenoidal approach

=One patient operated on by endoscopic Transplanum approach

Two patients died following an injury of the ICA

Post-operative morbidity consisted of: transient diabetes insipidus which ceased within 4 days. Transient Hemiparesis with fronto temporal infarction and CSF fistula in one patient. Surgical resection was complete in $45 \%$ of patients, subtotal and partial in $55 \%$.
\end{abstract}

Keywords: meningioma, surgery, tuberculum sellae, total excision, visual function.

\section{Introduction}

Tuberculum sellae meningiomas are benign tumors originating from the tuberculum sellae, limbus and chiasmatic sulcus. The first case of tuberculum sellae meningioma was reported by Stewart in 1899 when doing an ordinary autopsy. Cushing succeeded to perform the first total excision in 1916(2,6). In 1938, Cushing and Eisenhardt published a series of 24 cases of tuberculum sellae meningiomas and proposed a classification into four types following their size $(6,12)$. Many reports have been published in the literature concerning suprasellar meningiomas that helped clear the confusion between these and the tuberculum sellae meningiomas. Tuberculum sellae meningiomas represent between 3 and $10 \%$ of intracranial meningiomas $(2,6,7,11,12)$ .Their clinical symptomatology is primarily visual, well described by CUSHING in his monography. Their uniqueness lies in their strict median development, they are practically sitting on top of the tubercle and on the sellar diaphragm between the optic nerves and chiasma posteriorly, in contact with the pituitary stalk and anterior parts of the circle of Willis thus making theirsurgical resection difficult. In this paper, we report a clinicalstudy, radiological, surgical and visualoutcome of a series of 64cases operatedin 10 years.

\section{Patients And Methodes}

This is a retrospective study of 64casesof tuberculum sellar meningiomas operated in Ali Ait Idir hospital between 2005-2015, the average age of patients was 55,5years. The sex ratio was 2 females $/ 01$ male. The preoperative duration of symptoms varied between 02months-07years.Clinical signs were dominated by the opto-chiasmatic syndrome with visual disorders in $95 \%$. The unilateraldecrease in visual acuity was the mainsymptomin $40 \%$ and bilateral in $60 \%$ of cases. The incidence of blindness was noted to be significant $(40 \%)$.Visual field defects were noted in lesions with compression of the optic chiasma and retro chiasmalextension (bitemporal hemianopia classic in 15 patients). headaches were rare and were present in only $22 \%$ of patients.Other clinical symptom was diabetes insipidus in $11 \%$ of cases.

Most of our patients underwent a brain ct-scan and brain MRI with MR angiography, cerebral angiography was performed in one patient because of the extent of the tumor and the close relationship with the two internal carotids.Endocrine function analysis is routinely performed in presence ofendocrinologicalsigns (diabetes insipidus)Surgical treatment, 62 patients were operated by transcranial approach:

- Unilateral Sub-frontalapproach in 15 cas $(23.4 \%)$.

- The frontotemporal approach in 35 cases (54.6\%).

- The sub- frontalwith supra-orbital skin incision in 12 cases (19\%).

One patient operated by transsphenoidal approach $(1.5 \%)$.

One patient operated by endoscopic transplanum approach (1.5\%) 
In six patients, the tumor had invaded the ipsilateral optic canal which limited the quality of resection, in all cases the intraductal residual tumor was left in place, which explains the visual deterioration in postoperative long-term outcomes.

\section{III.Results}

$\square$ surgicalresults:The surgical excision was complete in $45 \%$ of patients, subtotal and partial in $5 \%$.Subtotal excision was considered if residual tumorwas left in place in the optical canal or a fragment which adheres to the internal carotid artery or the pituitary stalk or the optic chiasma.In some cases, the dural attachment to the tuberculum sellar was not removed, in all patients, however, the meningeal layer was well coagulated with bipolar cautery.

$\square$ visual Results:The visual function analysis was first performed in the immediate postoperative period looking for a visual improvement/ worsening by the neurosurgeon himself, followed by an ophthalmologist in the short and long term. Note the improvement in visual function in $45 \%$ of cases, remained unchanged in $40 \%$ of cases and worsened in $15 \%$ of patients. Visual field deficits were also improved after surgery.Ten patients were documented to have improvement in their preoperative visual field defect.

$\square$ postoperative follow up:All patients were regularly followed up clinically and radiologically by the neurosurgeon and ophthalmologist.The monitoring period is shorter varying froml year to 11,5 years. All patients with residual tumor underwent radiological examinations especially brain MRI to see the evolutionary potential of the lesion and for making treatment decisions. Note that in two patients, where the ipsilateral optic canal was invaded by the residual tumor left in place during surgery, the tumor grew and caused visual worsening thereafter, a consultation was requested for a possible radio surgery because of the close relationship of the tumor with the optic nerves, radio surgery was recommended.

Conformal radiation therapy has been proposed for both cases.In one patient, a residual tumor was followed for three years until the visual aggravation, the patient was operated on the same side of the first intervention, and recovered the visual function postoperatively.For patients where the quality of resection was satisfactory, an MRI was performed at 6-month intervals for the first year, every year for 5 years, and every two years thereafter.

$\square$ the mortalityand morbidity.We deplore the death of two cases by injury of the internal carotid artery during surgery (...).Postoperative morbidity was dominated by;

-transient diabetes insipidus which disappeared after 4 days,

-A Motor deficit of the left side of the body which recovered later with a good functional rehabilitation following a frontotemporal hemorrhagic infarction confirmed by a brain scan.

-a CSF leak in the case operated by the endoscopic transplanum approach

\section{Discussion}

Tuberculum sellar meningiomas are classified with anterior skull base meningiomas. The average age of discovery is the fourth decade with a female predominance $(2,11,12)$. Holmes and Sargent described the chiasmal syndrome "as a primary optic atrophy withbitemporal visual field defects in adult patients who had tumors in the suprasellar region and specifically in the tuberculum sellar region" (6).

In 1938 CUSHING and EISENTHARDH operated 24 cases of TSMs and proposed a classification in 4 stages depending on the size $(2,6,7)$.Preoperative period between the first clinical sign and the diagnosis is relatively short, from months to years. George (6) on a series of 23 patients, found an average of 16.7 months. This shorter period is explained by the fact that the tumor develops in a narrow region between the optic nerves and the chiasma, the optical canals will be compressed quickly which will cause the patient to seek for consultation fairly quickly.

It is rare todiscover tuberculum sellarmeningiomas by chance because even with small tumors, there are already signs and symptoms ofcompression of optic canals.- The clinical symptomatology is primarily visual, well described by CUSHING in his monograph $(2,6.11)$.Due to the development of the tumor close to the optical canals, pituitary gland and pituitary stalk, it can cause a compressive effect:

\footnotetext{
* Visual disturbances dominated by the opto-chiasmatic syndrome in $95 \%$ of cases $(2,6.11)$ :

- Drop in visual acuitybilaterally often asymmetrical, where one side is more affected than the other. This phenomenon is all the more marked when the diagnosis is late.

- A visual field defect can of either a classical bitemporalhemianopsia, or quadranopsia.

- Examination of the fundus may show clear optic atrophy 75-90\% of cases (direct compression of the optic nerves). This atrophy may be unilateral or bilateral (2.6).

* Endocrine disorders of one or more hormonal lines in 5 to $10 \%$ of cases; but their frequency remains fairly rare.
} 
The complete visual assessment is required before and after surgery $(2,6)$, it includes the visual field examination, examination of the fundus and visual evoked potentials. The endocrine function analysis is also essential (full hypophysiogramme).Imaging study (CT, MRI and angiography sometimes) preoperatively is important to appreciate exactly; the site of the tumor, the extent and significance of the insertion, relationof the tumor with the optical canals, the pituitary stalk, pituitary and anterior elements of the circle of Willis base to choose the ideal approach. These images clearly define the three dimensional measurement of the tumorand its relationship with the cavernous sinus, optic chiasm, hypothalamus, and major cerebral arteries. Themeningiomas are iso intense on T1-weighted images and hypointense on T2 sequences.

MRI can often make the difference between tuberculum sellar meningioma and pituitary adenoma especially in sagittal sections where the analysis of the dural implementation of meningioma is properly seen, moreover the contrast enhancement of the base of insertion that extends to the dura juxtatumoral is very supportive of meningioma.

\section{Surgery:}

Currently, despite the close relationship of the tumor with the optical canals, anterior elements of the circle of Willis and the pituitary stalk, the rate of mortality and morbidity have decreased.

The preservation of visual function is the goal of treatment, Intraoperative key to preservation of visual function is to minimize the direct manipulation or trauma to the optic nerve and avoid the injury of arterial supply of the optical canals $(2,6,7.12)$. Moreover, during surgery, tumor excison starts with the reduction in tumor volume which should start from the center of the tumor, where no vital structures are present.

The surgical technique is dictated by the clinical study and especially preoperative imaging to define the relationship of the meningioma with vital structures, its volume, its extension, its vascularization and bone reactions.

Several approaches have been described for the surgical treatment of tuberculum sellarmeningioma ,in our series the following approaches were used:

- Unilateral Sub-frontalapproach in 15cases (23.4\%).

- The frontotemporal approach in 35 cases $(54.6 \%)$.

- The sub- frontalwith supra-orbital skin incision in 12 cases $(19 \%)$.

One patient operated by transsphenoidal approach $(1.5 \%)$.

One patient operated by endoscopic transplanum approach $(1.5 \%)$

Usually unilateral bone flap is enough. The choice of the approach depends on the habits of the surgeon and the extension of the tumor; some authors operate on the side where the visual acuity is the most diminished.

\section{* Theunilateral sub frontal approach:}

The patient is supine, head elevated 10 to $15^{\circ}$ above the shoulders, the head slightly extended to facilitate the separation of the frontal lobes (no rotation of the head).

The skin incision is arcuate from $1 \mathrm{~cm}$ in front of the tragus, backwards following the hairline to $2 \mathrm{~cm}$ beyond the midline. The scalp is folded forward with avulsion of the temporalis muscle, which is folded out.

Making a frontal bone flap, the most important hole is that located at the junction between the zygoma and the outer edge of the orbit, with help of thecraniotome. craniotomy is started parallel to the orbital roof toward the midline behind. Afree flap of 5 holes is cut with aGIGLI saw from flush orbit crossing the superior sagittal sinus and sylvianvalley.

In case of opening of the frontal sinus, a monopolar coagulation and resection of the sinus mucosa followed by obstructing thesinus with bone powder or bybetadinisedwax and then isolating sinus by folding back the scalp.It is imperative to change the instruments used in this phase.

- Other changes are made to this approach, such as resection of the orbital arcade proposed by ELMEFTY where craniotomy including the upper outer part of the orbit (upper outerorbitotomy), the front of the roof, the orbit and adjacent frontal bone.

After coagulation of the vessels of the dura in case of bleeding, the dural incision is concave forward with respect to subsequent revamps

\section{* Unilateral latero-frontal approach:}

The head is turned $30^{\circ}$ to the opposite side with a slight extension, skin incision much more limited, extending from the zygoma to the midline.

Craniotomy is made on the anterolateral aspect of the frontal bone using the first technique but extending less towards the midline.

The resection of pterion is performed

The incision of the dura is parallel to the base of the ACF 
* The pterional approach: it is an approach that has been proposed by DANDY and described by YASARGYL. It is much more lateral because the rotation of the head is greater between 50 and $60^{\circ}$

- The incision is similar to the fronto-lateral

- Craniotomy is centered on the pterion, extending from the temporal wing up the superiortemporal line and frontozygomaticsuture.

- The dura is detached from the wing of the sphenoid which is resected to its middlethird.

\section{* The supraorbital subfrontal approach:}

The lesions that aremedially located and not very bigvolumecan be tackled by a minimally invasive technique known as a unilateral sub frontal supraorbital eyebrow incision.

The incision is made at the upper limit of the eyebrow, from its inner portion above up to the outer orbital curvature. The vertical part of the frontal bone and the orbital arcade are disengaged, the peri-orbit is detached from the roof of the orbit. A bone flap using the oscillating saw carrying the orbital arcade off the orbital roof.

The incision of the dura mater is rectilinear parallel to the orbital plane of the arch with two lower discharge lines.The frontal lobe is retracted up progressively towards the basal cisterns, allowing the aspiration of CSF and brain relaxation. The procedure is then carried out conventionally, by piecemealexcision.

The closing time is easy is fast; sealed closure of the dura mater is achieved by separate or continoussuturing. Replacing the bone flap, the drill hole is filled by the bone powder. Reconstructing the muscular planes by separate knots, finally closing skin by intradermal knots or running suture.

\section{1 - reduction in tumor volume \\ 2- coagulation of feeder vessels \\ 3- dissection of the capsule from adjacent structures}

Whatever the route used intraoperatively 03 goals are to be considered:

The first description of the transsphenoidal approach for supra sellar tumorswas made by Weiss in 1987, several series have been reported in literature $(2,4,7.8)$ :

Kaptain et al JNS 2001

KIM et al JNS 2000

Kitano and Kaneda JNS 2001.

$\square$ JHON et al JNS 2003.

Currently with the development of minimally invasive surgery, endoscopic endonasal approach has become an excellent alternative for TSM surgery and other lesions of the skull base.

Sphenoid planum meningiomas or tuberculum sellae are the indication of choice for an extended endoscopic approach, due to their median location and small size (generally pre-chiasmatic).Access to the suprasellar region and the sphenoid planum is obtained through a more anterior trajectory than that of the sellar region.This approach allows the surgeon to make a significant intra capsular tumor reduction,optic nerve decompressionand an initial devascularization of the dura at the beginning of the intervention.In addition, this approach offers the possibility of excision of not only the tumor but also the dura and invadedbone.

\section{The visual prognosis:}

It has been shown that several factors influence the visual prognosis. Multiple authors have tried to define the prognostic factors of visual function:

1- influence of duration of visual symptoms preoperatively:

- According SIMON series of 101 cases, improved visual functions were observed in 50\% for a period of visual symptoms $<2$ years compared to $32 \%$ in patients whose clinical history exceeds 02 years (4) .

- As for ANDREWS WILSON and a series of 38 cases JNS1988 visual acuity and postoperative visual field were better in patients whose symptoms progressed for less than 06 months $(1,3,4)$.

- In the series of FAHLBUSH and SCHOTT 70 cases in 2002 (5);

$100 \%$ improvement for patients whose symptoms progressed for less than 6 months

$83 \%$ improvement for patients whose symptoms progressed of $6-12$ months

$60 \%$ improvement for patients whose symptoms developed over 12 months

\section{2- tumor volume:}

Is considered very important, it appears that when it exceeds $3 \mathrm{~cm}$ visual prognosis after surgery is jeopardized (JNS SKAR 2000) and depends on the tumor spread to the sellae diaphragm and anterior clinoid, cavernous sinus, orbit and sellarturcica. 
- It is no longer considered that it is the effect of direct compression of the optic nerves but that of thesmall vessels that vascularize them (post ethmoid artery, suppituitary artery ACA)

3-degree of loss of visual acuity preoperatively:

All authors (GOEL2002, JALLO2002, RACO 1999) agree that a preoperative visual acuity reduced very little chance of recovery after surgery, recovery is possible but not in the immediate postoperative period.

Deterioration in this case is due to the manipulation of previously fragile optical canals.

Optic atrophy is poor prognosis.

3 other factors:

- Some authors have reported age as a predictor

Rubin For Acta 199467 Cases Limited Age Is 54 Years.

For Zevgaridisacta 200162 cases the age limit 64 years.

- Invasion of optical canal

$\S$ In summary predictors of postoperative visual acuity are:

- preoperatively:

- Duration of symptoms $<6$ months

- Slightly reduced preoperative visual acuity

- Optic atrophy on fundoscopy

Operative mortality in tuberculumsellaeandsuprasellarmeningiomas varies widely in published series. It varies from 0 to $67 \%$ according to the authors

(6) .The highest figures correspond to the older series.

Currently the mortality figures rarely exceed $20 \%(1,3,4,6.10)$. The operative complications are few, usually, dominated mostly byvisual aggravation ranging from 10 to $20 \%$ depending on the series $(2,6.7)$ epilepsy, endocrine disorders, L.C.R fistula, infections, anosmia and diabetes insipidus.The quality of resection depends on the tumor size, the extent of the insertion base, the extension of the tumor and the relationship of the lesion with the neighboring arteries (carotid and anterior cerebral) when taken in the tumor resection is rarely total.

Conventional radiotherapy or radiosurgery are reserved for patients in whom resection could not be total, and in case of recurrence.
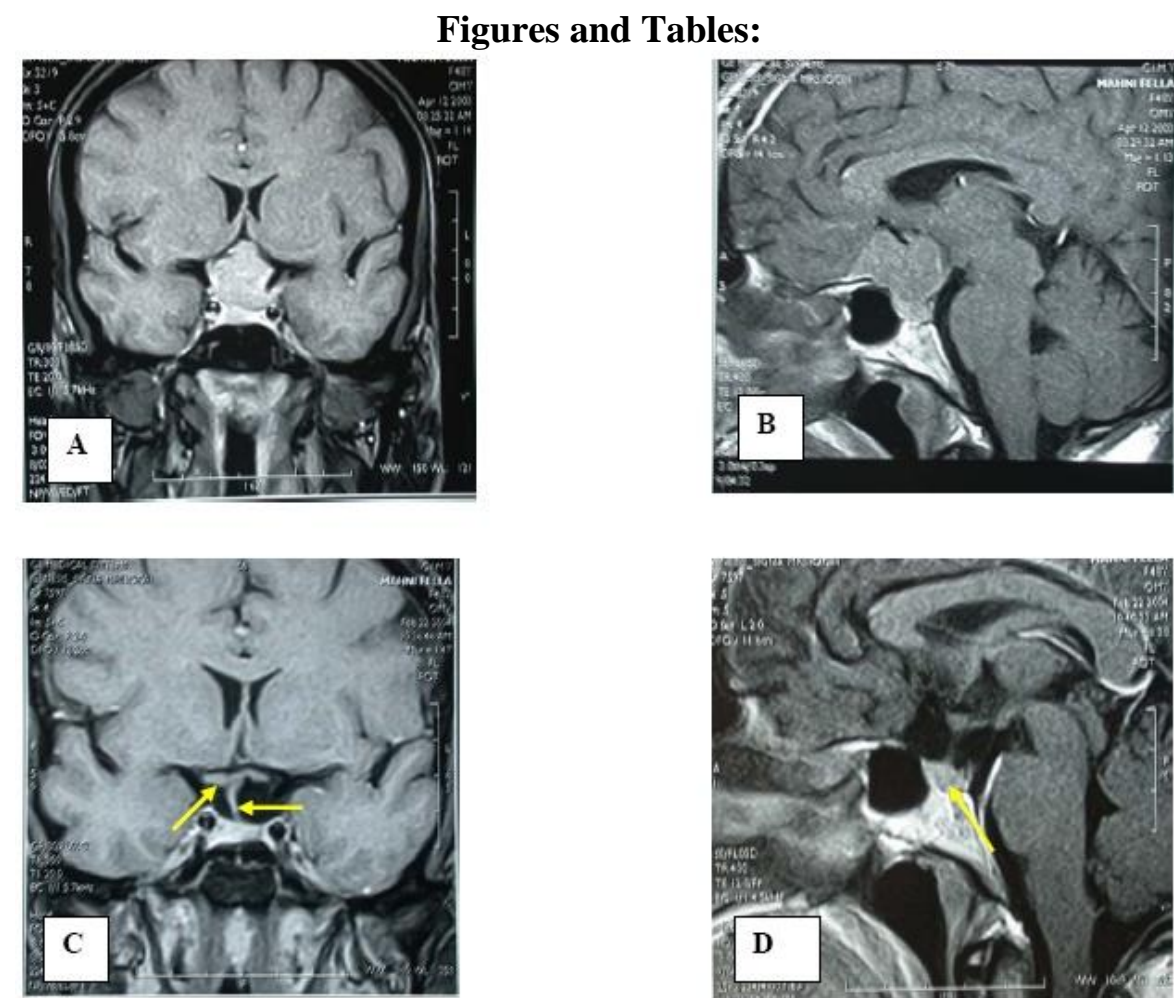

Fig1 :preoperative Post operative and MRI which shows the quality of excision of tuberculum sellar meningioma with preservation of the pituitary stalk. 


\section{Conclusion}

Tuberculum sellae meningiomas should be resected as soon as possible in patients with visual symptoms.A preoperative evaluation is necessary to give anobjectivepostoperative visual assessment after surgical resection. The total resection of meningiomas is the only guarantee of a cure.

\section{References}

[1]. Al Mefty O, Holoubi A, Rifai A, Fox JL :Microsurgicalremoval of suprasellar meningiomas.Neurosurgery 16:364-72,1985.

[2]. Chi JH, McDermott MW :Tuberculumsellaemeningiomas.Neurosurg Focus14(6) :Article 6,2003.

[3]. Ciric I, Rosenblatt S :Suprasellarmeningiomas(operative nuances).Neurosurgery 49:1372-77,2001.

[4]. Cook S, Smith Z, Kelly D:EndonasalTranssphenoidal Removal of Tuberculum Sellae Meningiomas.

[5]. Neurosurgery 55 (1):239-246, 2004.

[6]. Fahlbusch R, Schott W: Pterional surgery of meningiomas of the tuberculum sellae and planum sphenoidale : surgical results with special consideration of ophtalmological and endocrinological

[7]. Outcomes .Jneurosurg 96:235-243,2002.

[8]. George J, ValloB :Tuberculumsellaemeningiomas :Microsurgical anatomy and Surgical technique.Neurosurgery 51:1432-40,2002.

[9]. Goel A, Muzumdar D, Desai K :Tuberculum sellae meningiomas :A reort on management on the basis of a surgical experience with 70 patients.Neurosurgery 51:1358-64,2002.

[10]. Jho H, Park I, Kim M:EndoscopicEndonsale Cranial Base Surgery.(Abstract of oral presentations) .Neurosurgery 49 (2):524525,2001 .

[11]. Lindley JG, Challa VR, Kelly DL :Meningiomas and brain edema.in Al-Mefty O(ed): Meningiomas.New York,Raven,1991,pp5973.

[12]. Morsli A, Ait Bachir M, Ounnoughene M, et al :Les méningiomes du tubercule de la selle turcique :Apropos de 14 cas. JSFNC2014.

[13]. Philippon J : Les méningiomes.Dans Philippon J : Les tumeurs cerebrales du diagnostic au traitement ,MASSON Paris 2004 ,pp169-179.

[14]. Vallo Benjamin, M.D.Stephen M. Russell, M.D :the microsurgical nuances of resecting tuberculum sellae meningiomas,neurosurgery 56[onssuppl 2]:ons-411-ons-417, 2005 